\title{
REFLECTION OF P-WAVE AND SV-WAVE IN A GENERALIZED TWO TEMPERATURE THERMOELASTIC HALF-SPACE
}

\author{
S. SANTRA \\ Department of Mathematics \\ Gargi Memorial Institute of Technology \\ Kolkata-700144, INDIA \\ E-mail: sutapasantra.ismu.math@gmail.com \\ A. LAHIRI ${ }^{*}$ and N.C. DAS \\ Department of Mathematics \\ Jadavpur University \\ Kolkata-700032, INDIA \\ E-mails: Lahiriabhijit2000@yahoo.com \\ ncdasmaths@gmail.com
}

\begin{abstract}
In this work the theory of two temperature generalized thermoelasticity has been used to investigate the problem of reflection of P-wave and SV-wave in a half space when the surface is i) thermally insulated or ii) isothermal. The ratios of the reflection coefficient to that of the incident coefficient for different cases are obtained for P-wave and SV-waves. The results for various cases for the conductive and dynamical temperature have been compared. The results arrived at in the absence of the thermal field (elastic case) have also been compared with those in the existing literature. Finally, the results for various cases have been analyzed and depicted in graphs.
\end{abstract}

Key words: P-wave, SV-wave, conductive temperature, dynamical temperature, second sound.

\section{Introduction}

In the classical uncoupled and coupled theory of thermoelasticity, the heat transport equation involved is of diffusion type which predicts infinite speed of propagation of thermal signals, whereas the equation of motion is of hyperbolic type and as such the elastic disturbance propagates with finite speed. The non-classical theory of thermolasticity involves hyperbolic type of equations in both the heat conduction equation and mechanical equations of motion.

At present, there are three generalizations of the thermoelastic theory which are supported by experiments, revealing the actual occurrence of wave type heat flow in solids. Suhubi (1982) called this phenomenon the second sound effect, the first sound being the usual sound wave. Lord and Shulman (1967) employed a modified version of the Fourier law of heat conduction by introducing a flux-rate term and thereby established the generalized version of the heat transport equation which is hyperbolic in nature.

In the L-S (Lord and Shulman, 1967) theory only one relaxation parameter having the dimension of time has been used. Later Green and Lindsay (1972) presented a theory of thermoelasticity with certain special features that contrast with the L-S theory. In the G-L model, the Fourier law of heat conduction is unchanged whereas the constitutive relations and the classical energy equation are modified with two time relaxation parameters. The third generalization of the theory was proposed by Green and Nagdhi (1992; 1993 ; 1996) who provide sufficient basic modifications in the constitutive equations that permit solution of a

\footnotetext{
* To whom correspondence should be addressed
} 
much wider class of heat flow problems. These theories also provide finite speed of propagation of heat waves.

Chen and Gurtin (1968a) and Chen et al. (1968b) formulated a theory of heat conduction in deformable bodies, which depends on two distinct temperatures, the conductive temperature $\chi$ and the thermodynamic temperature $T$. For time dependent problems and for wave propagation problems in particular, the two temperatures are in general different, regardless of the presence of heat supply. The basic equations of two temperature generalized thermoelastic theory have been deduced by Youssef (2006). The problem of two temperature disturbances in an infinite body with a spherical cavity subjected to different types of thermal loading was solved by Youssef et al. (2007). Based on two temperature generalized theory Bassiouny et al. (2008) solved the problem of a thermopiezoelectric rod under different types of thermal loading. Das et al. (2008) solved the problem of reflection of generalized thermoelastic waves on a half space using the GN-model (1993).

So far as the present authors are aware, no work on reflection of waves on the boundary of a half space based on two temperature theory has been done. We now propose to work on the problem the reflection of P- and SV- waves in a rigidly fixed isothermal or thermally insulated boundary. The amplitude ratios for different cases have been calculated and the results are depicted in several graphs showing the nature of variations due to conductive and dynamic temperature.

\section{Formulation of the problem and solution}

Let the origin of the rectangular Cartesian coordinate system be fixed on the boundary of the homogeneous, isotropic elastic half space with the $z>0$ axis directed normally inside the medium with the $x$-axis along the horizontal direction. The axis of $y$ is taken in the direction of the line of intersection of the plane wave front with the plane surface Fig.1.

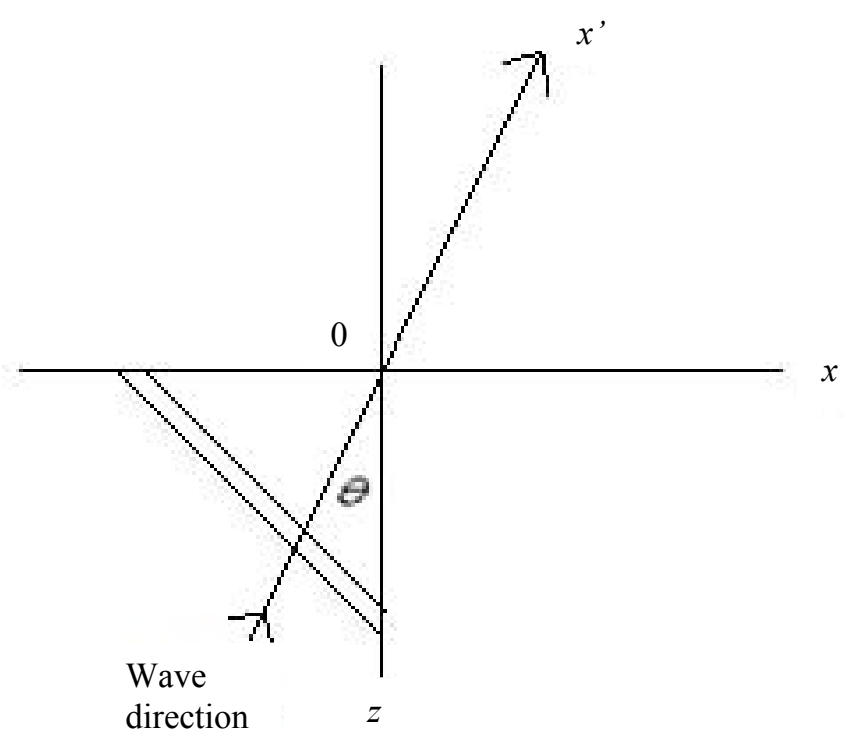

Fig.1. Intersection of the wave front by $x z$-plane.

If we restrict our analysis to plane strain in the $x z$-plane, then all the field variables may be taken as a function of $x, z$ and $t$. Then the displacement vector $\boldsymbol{u}$, the thermodynamical temperature $T$, and the conductive temperature $\chi$ may be taken as 


$$
\boldsymbol{u}=[u(x, z, t), 0, w(x, z, t)], \quad T=T(x, z, t), \quad \text { and } \quad \chi=\chi(x, z, t)
$$

Following the L-S model of generalized thermoelasticity, the equations of motion for an isotropic elastic solid in the absence of body force, the heat conduction equation in the absence of heat source may be written as in Youssef (2006)

$$
\begin{aligned}
& (\lambda+\mu) \nabla(\nabla \cdot \boldsymbol{u})+\mu \nabla^{2} \boldsymbol{u}-\gamma \nabla \cdot T=\rho \ddot{\boldsymbol{u}}, \\
& k \chi_{, i i}=\rho c_{E}\left(\dot{T}+\tau_{0} \ddot{T}\right)+\gamma T_{0}\left(\dot{e}_{k k}+\tau_{0} \ddot{e}_{k k}\right)
\end{aligned}
$$

where the relation

$$
\chi-T=a \nabla^{2} \chi, \quad a>0,
$$

is to be satisfied by the conductive temperature $\chi$ and thermodynamic temperature $T$. Here ' $a$ ' is called the two temperature parameter.

The stress-strain relation may be written as

$$
\tau_{i j}=\lambda u_{k, k} \delta_{i j}+\mu\left(u_{i, j}+u_{j, i}\right)-\gamma T
$$

where $\lambda$ and $\mu$ are Lame constants, $\gamma=(3 \lambda+2 \mu) \alpha_{t}, \alpha_{t}$ being the coefficient of thermal expansion, $\rho$ is the mass density, $k$ is the thermal conductivity, $c_{E}$ is the specific heat at constant strain, $T_{0}$ is the reference temperature, and $\tau_{0}$ is the relaxation time parameter, the system of Eqs (2.1) and (2.2) are hyperbolic in nature and as such both the elastic and thermal disturbance propagate in the medium with finite speed.

In order to non-dimensionalize the equations, we define the following quantities

$$
\begin{array}{lll}
x^{\prime}=\frac{w^{*} x}{c_{1}}, & z^{\prime}=\frac{w^{*} z}{c_{1}}, & t^{\prime}=\frac{w^{*} t}{c_{1}}, \\
\chi^{\prime}=\frac{\chi}{T_{0}}, & a^{\prime}=\frac{a}{T_{0}}, & u^{\prime}=\frac{\rho w^{*} c_{1} u}{\gamma T_{0}}, \quad w^{\prime}=\frac{\rho w^{*} c_{1} w}{\gamma T_{0}}, \\
\tau_{i j}{ }^{\prime}=\frac{\tau_{i j}}{\gamma T_{0}}, & \tau_{0}{ }^{\prime}=w^{*} \tau_{0}, & w^{*}=\frac{c_{E}(\lambda+2 \mu)}{k} .
\end{array}
$$

Suppressing primes for convenience in the notation, the dimensionless forms of Eqs (2.1)-(2.4) will be as follows

$$
\begin{aligned}
& c_{2}^{2} \nabla^{2} \boldsymbol{u}+\left(c_{1}^{2}-c_{2}^{2}\right) \nabla(\nabla \cdot \boldsymbol{u})-c_{I}^{2} \nabla \cdot T=c_{l}^{2} \ddot{\boldsymbol{u}}, \\
& \nabla^{2} \chi=\dot{T}+\tau_{0} \ddot{T}+\varepsilon\left(\nabla \cdot \dot{\boldsymbol{u}}+\tau_{0} \nabla \cdot \ddot{\boldsymbol{u}}\right),
\end{aligned}
$$




$$
\begin{aligned}
& \chi-T=a\left\{\dot{T}+\tau_{0} \ddot{T}+\varepsilon\left(\nabla \cdot \dot{\boldsymbol{u}}+\tau_{0} \nabla \cdot \ddot{\boldsymbol{u}}\right)\right\} \\
& \tau_{i j}=\left(1-\frac{2 c_{2}^{2}}{c_{1}^{2}}\right)(\operatorname{div} \boldsymbol{u}) \delta_{i j}+\frac{2 c_{2}^{2}}{c_{1}^{2}} e_{i j}-T \delta_{i j}
\end{aligned}
$$

where, $\quad c_{1}^{2}=\frac{\lambda+2 \mu}{\rho}, \quad c_{2}^{2}=\frac{\mu}{\rho}, \quad \delta^{2}=\frac{c_{2}^{2}}{c_{1}^{2}}, \quad \varepsilon=\frac{\gamma^{2} T_{0}}{\rho c_{E}(\lambda+2 \mu)}$.

Let us consider a decomposition of the displacement vector in the form

$$
\boldsymbol{u}=\operatorname{grad} \phi+\operatorname{curl} \psi, \quad \operatorname{div} \psi=0
$$

where $\phi$ and $\psi$ are thermoelastic potential functions. Substituting Eq.(2.10) in Eqs (2.6)-(2.7), we obtain

$$
\begin{aligned}
& c_{1}^{2} \nabla\left[\nabla^{2} \phi-\left(1-a \nabla^{2}\right) \chi-\ddot{\phi}\right]+\nabla \times\left[c_{2}^{2} \nabla^{2} \psi-c_{1}^{2} \ddot{\psi}\right]=0, \\
& \nabla^{2} \chi-\left(1-a \nabla^{2}\right)\left(\dot{\chi}+\tau_{0} \ddot{\chi}\right)=\varepsilon \nabla^{2}\left(\dot{\varphi}+\tau_{0} \ddot{\phi}\right) .
\end{aligned}
$$

Equation (2.11) will be satisfied if

$$
\nabla^{2} \phi-\ddot{\phi}=\left(1-a \nabla^{2}\right) \chi
$$

and

$$
\nabla^{2} \psi-\frac{1}{\delta^{2}} \ddot{\psi}=0
$$

From Eqs (2.13) and (2.14) we see that the P-wave is affected in the presence of the thermal field while SV-wave remains unaffected.

Eliminating $\chi$ from Eqs (2.12) and (2.13), we get the equation for the thermoelastic potential function $\phi$ as

$$
\begin{aligned}
& \nabla^{4} \phi-(1+\varepsilon)\left(1-a \nabla^{2}\right) \nabla^{2} \dot{\phi}-[1+(1+\varepsilon)] \tau_{0}\left(1-a \nabla^{2}\right) \nabla^{2} \ddot{\phi}+ \\
& +\left(1-a \nabla^{2}\right) \dddot{\phi}+\tau_{0}\left(1-a \nabla^{2}\right) \dddot{\phi}=0 .
\end{aligned}
$$

Choosing $\psi=(0, \psi, 0)$, Eq. (2.14) takes the form

$$
\nabla^{2} \psi=\frac{1}{\delta^{2}} \ddot{\psi}
$$

Thus the potential $\psi$ corresponds to the displacement in the $x z$-plane due to SV-wave. The displacement and stress components in terms of the potential function can be written as 


$$
\begin{aligned}
& u=\frac{\partial \phi}{\partial x}-\frac{\partial \psi}{\partial z}, \quad v=0, \quad w=\frac{\partial \phi}{\partial z}+\frac{\partial \psi}{\partial x} \\
& \tau_{z z}=\left(\frac{\partial^{2} \phi}{\partial x^{2}}-\frac{\partial^{2} \psi}{\partial z^{2}}\right)+\frac{2 c_{2}^{2}}{c_{1}^{2}}\left(\frac{\partial^{2} \psi}{\partial x \partial z}-\frac{\partial^{2} \phi}{\partial x^{2}}\right)-\left(1-a \nabla^{2}\right) \chi \\
& \tau_{z x}=\frac{c_{2}^{2}}{c_{1}^{2}}\left(2 \frac{\partial^{2} \phi}{\partial x \partial z}+\frac{\partial^{2} \psi}{\partial x^{2}}-\frac{\partial^{2} \psi}{\partial z^{2}}\right) .
\end{aligned}
$$

For plane P-wave propagating in the $x^{\prime}$-direction (as in Fig.1) we have

$$
\phi=A e^{i\left(k x^{\prime}-\omega t\right)}
$$

where $k$ is the wave number and $\omega$ is the circular frequency of the wave. Now $x^{\prime}$ can be calculated as

$$
x^{\prime}=x \sin \theta-z \cos \theta
$$

where $\theta$ is the angle of inclination of the incident wave with the normal to the surface. Thus $\phi$ becomes

$$
\phi=A e^{i\{k(x \sin \theta-z \cos \theta)-\omega t\}} .
$$

Substituting Eq.(2.18) in Eq.(2.15), we get a quadratic equation in $k^{2}$ as

$$
\begin{aligned}
& k^{4}\left[1-a\left\{(1+\varepsilon)\left(i \omega+\tau_{0} \omega^{2}\right)+\omega^{2}\right\}\right]-k^{2}\left[\left(1+\varepsilon-a \omega^{2}\right)\left(i \omega+\tau_{0} \omega^{2}\right)+\omega^{2}\right]+ \\
& +\omega^{2}\left(i \omega+\tau_{0} \omega^{2}\right)=0 .
\end{aligned}
$$

Solving this Eq.(2.20), the roots of this equation may be written as

$$
\begin{array}{lc}
k_{1}^{2}=M_{1}+i N_{1}, & k_{2}^{2}=M_{2}+i N_{2}, \\
M_{1}=\omega^{2}\left\{1+\frac{\omega^{4} a-\varepsilon}{\omega^{2}+1}\right\}, & N_{1}=\frac{\omega^{3}\left(a \omega^{2}+\varepsilon\right)}{\omega^{2}+1}, \\
M_{2}=\omega^{2}\left\{\tau_{0}-\frac{a-\varepsilon}{\omega^{2}+1}\right\}, & N_{2}=\omega\left\{1-a+\frac{a+\varepsilon}{\omega^{2}+1}\right\}
\end{array}
$$

In the same way we can also write down the solution of Eq.(2.16) as

$$
\psi=B e^{i\left\{k_{3}(x \sin \theta-z \cos \theta)-\omega t\right\}}
$$


where, $\quad k_{3}^{2}=\frac{\omega^{2}}{\delta^{2}}$.

\section{CASE I: Plane P-wave incident upon a plane surface}

Let the angle of incidence with the normal to the surface be $\theta_{0}$ (Fig.2). Then for P-wave, we may take the following forms

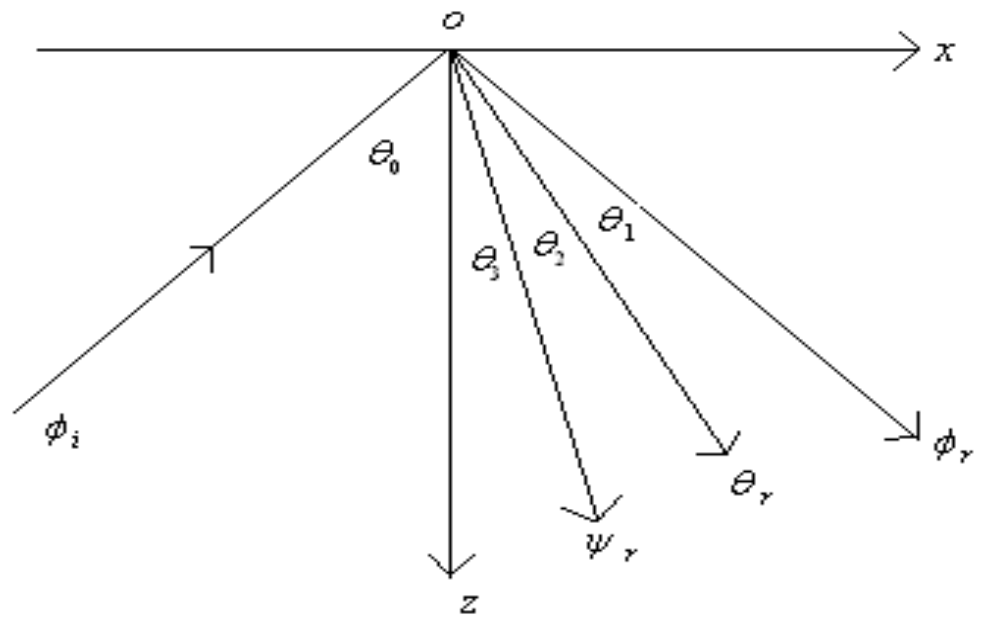

Fig.2. Incident P-wave and corresponding reflected waves.

$$
\begin{aligned}
& \phi_{i}=A_{3} e^{i k_{l}\left(x \sin \theta_{0}-z \cos \theta_{0}\right)}, \\
& \phi_{r}=A_{4} e^{i k_{l}\left(x \sin \theta_{1}+z \cos \theta_{1}\right)}+A_{2} e^{i k_{2}\left(x \sin \theta_{2}+z \cos \theta_{2}\right)}, \\
& \psi_{r}=A_{6} e^{i k_{3}\left(x \sin \theta_{3}+z \cos \theta_{3}\right)}
\end{aligned}
$$

where, the suffix $i$ and $r$ correspond to incident and reflected waves. The term $e^{-i \omega t}$ has been omitted from Eqs (2.24)-(2.26) for simplicity.

Since $\phi=\phi_{i}+\phi_{r}$, so $\chi_{r}$ can now be calculated from Eq.(2.13) as

$$
\left(1-a \nabla^{2}\right) \chi_{r}=b_{1} A_{2} e^{i k_{2}\left(x \sin \theta_{2}+z \cos \theta_{2}\right)}+b_{2} A_{3} e^{i k_{l}\left(x \sin \theta_{0}-z \cos \theta_{0}\right)}+b_{3} A_{4} e^{i k_{l}\left(x \sin \theta_{l}+z \cos \theta_{l}\right)}
$$

where

$$
b_{1}=\omega^{2}-k_{2}^{2}, \quad b_{2}=b_{3}=\omega^{2}-k_{1}^{2} .
$$

The components of displacements and stresses can be calculated from Eq.(2.17) by changing

$$
\phi=\phi_{i}+\phi_{r}, \quad \psi=\psi_{r}, \quad \text { and } \quad \chi=\chi_{r} .
$$




\section{$\underline{\text { Reflection at rigid boundary }}$}

The boundary conditions are given by

$$
u=w=\frac{\partial T}{\partial z}+h T=0, \quad \text { on } \quad z=0
$$

where $h \rightarrow 0$ and $h \rightarrow \infty$, correspond to thermally insulated and isothermal boundaries respectively.

Using Eqs (2.24)-(2.28), we calculate the displacement from Eq.(2.17) and then using the boundary condition Eq.(2.29), we obtain

$$
\begin{aligned}
& i k_{1} \sin \theta_{0} A_{3} e^{i k_{1} x \sin \theta_{0}}+i k_{1} \sin \theta_{1} A_{4} e^{i k_{1} x \sin \theta_{1}}+ \\
& +i k_{2} \sin \theta_{2} A_{2} e^{i k_{2} x \sin \theta_{2}}-i k_{3} \cos \theta_{3} A_{6} e^{i k_{3} x \sin \theta_{3}}=0, \\
& -i k_{1} \cos \theta_{0} A_{3} e^{i k_{1} x \sin \theta_{0}}+i k_{1} \cos \theta_{1} A_{4} e^{i k_{1} x \sin \theta_{1}}+ \\
& +i k_{2} \cos \theta_{2} A_{2} e^{i k_{2} x \sin \theta_{2}}+i k_{3} \sin \theta_{3} A_{6} e^{i k_{3} x \sin \theta_{3}}=0, \\
& i\left[b_{1} A_{2} k_{2} \cos \theta_{2} e^{i k_{2} x \sin \theta_{2}}-b_{2} A_{3} k_{1} \cos \theta_{0} e^{i k_{1} x \sin \theta_{0}}+b_{2} A_{4} k_{1} \cos \theta_{1} e^{i k_{1} x \sin \theta_{1}}\right]+ \\
& +h\left[b_{1} A_{2} e^{i k_{2} x \sin \theta_{2}}+b_{2} A_{3} e^{i k_{1} x \sin \theta_{0}}+b_{2} A_{4} e^{i k_{1} x \sin \theta_{1}}\right]=0 .
\end{aligned}
$$

In order that Eqs (2.30)-(2.32) might hold for all values of $x$, the terms in the left hand side should be independent of $x$, and this condition is satisfied if

$$
k_{1} \sin \theta_{0}=k_{1} \sin \theta_{1}=k_{2} \sin \theta_{2}=k_{3} \sin \theta_{3} .
$$

Equation (2.33) implies that

$$
\begin{aligned}
& \sin \theta_{0}=\sin \theta_{1}=\frac{k_{2}}{k_{1}} \sin \theta_{2}=\frac{k_{3}}{k_{1}} \sin \theta_{3}, \\
& \sin \theta_{0}=\sin \theta_{1} \Rightarrow \theta_{0}=\theta_{1} .
\end{aligned}
$$

In the case $\varepsilon=\tau_{0}=0$, we get

$$
\theta_{0}=\theta_{1} \quad \text { and } \quad \frac{\sin \theta_{0}}{c_{1}}=\frac{\sin \theta_{3}}{c_{2}}
$$

which is Snell's law.

Using Eq.(2.33) in Eqs (2.30)-(2.32) we get three linear homogeneous equations for the four unknowns $A_{2}, A_{3}, A_{4}$ and $A_{6}$. Hence we prefer to calculate the amplitude ratios $\frac{A_{2}}{A_{3}}, \frac{A_{4}}{A_{3}}$ and $\frac{A_{6}}{A_{3}}$ of the waves by applying different boundary conditions. 


\section{$\underline{\text { Incident P-wave at insulated rigid boundary }}(h \rightarrow 0)$}

Equations (2.30)-(2.32), after reviewing through conditions Eqs (2.33) and (2.34) and taking $h \rightarrow 0$, may be written in the form

$$
\left[\begin{array}{ccc}
a_{11} & a_{12} & a_{13} \\
a_{21} & a_{22} & a_{23} \\
a_{31} & a_{32} & 0
\end{array}\right]\left[\begin{array}{l}
A_{2} / A_{3} \\
A_{4} / A_{3} \\
A_{6} / A_{3}
\end{array}\right]=\left[\begin{array}{c}
-a_{12} \\
a_{22} \\
a_{32}
\end{array}\right]
$$

where

$$
\begin{array}{lll}
a_{11}=\frac{k_{2}}{k_{1}} \sin \theta_{2}, & a_{12}=\sin \theta_{0}, & a_{13}=-\frac{k_{3}}{k_{1}} \cos \theta_{3}, \\
a_{21}=\frac{k_{2}}{k_{1}} \cos \theta_{2}, & a_{22}=\cos \theta_{0}, & a_{23}=\frac{k_{3}}{k_{1}} \sin \theta_{3}, \\
a_{31}=\frac{k_{2}^{3}}{k_{1}^{3}}\left(\frac{\omega^{2}}{k_{2}^{2}}-1\right) \cos \theta_{2}, & a_{32}=\left(\frac{\omega^{2}}{k_{1}^{2}}-1\right) \cos \theta_{0} .
\end{array}
$$

Solving Eq.(2.36), we get

$$
\begin{aligned}
\frac{A_{2}}{A_{3}} & =\frac{1}{\Delta}\left(\frac{\omega^{2}}{k_{1}^{2}}-1\right) \frac{k_{3}}{k_{1}} \sin 2 \theta_{0} \sin \theta_{3}, \\
\frac{A_{4}}{A_{3}} & =1-\frac{2}{\Delta} \frac{k_{2}^{3}}{k_{1}^{3}} \frac{k_{3}}{k_{1}}\left(\frac{\omega^{2}}{k_{2}^{2}}-1\right) \cos \theta_{2} \sin \theta_{0} \sin \theta_{3}, \\
\text { where } \quad \frac{A_{6}}{A_{3}} & =\frac{1}{\Delta} \frac{k_{2}}{k_{1}}\left(1-\frac{k_{2}^{2}}{k_{1}^{2}}\right) \cos \theta_{2} \sin 2 \theta_{0} \\
\Delta & =\left\{\frac{k_{2}}{k_{1}}\left(\frac{\omega^{2}}{k_{1}^{2}}-1\right) \cos \theta_{0} \sin \theta_{2}-\frac{k_{2}^{3}}{k_{1}^{3}}\left(\frac{\omega^{2}}{k_{2}^{2}}-1\right) \cos \theta_{2} \sin \theta_{1}\right\} \frac{k_{3}}{k_{1}} \sin \theta_{3}+ \\
+ & \left\{\frac{k_{2}}{k_{1}}\left(\frac{\omega^{2}}{k_{1}^{2}}-1\right) \cos \theta_{0} \cos \theta_{2}-\frac{k_{2}^{3}}{k_{1}^{3}}\left(\frac{\omega^{2}}{k_{2}^{2}}-1\right) \cos \theta_{2} \cos \theta_{1}\right\} \frac{k_{3}}{k_{1}} \cos \theta_{3} .
\end{aligned}
$$

In the case when $\varepsilon=\tau_{0}=a=0$, Eqs (2.38)-(2.40) take the form

$$
\begin{aligned}
& \frac{A_{2}}{A_{3}}=0, \quad \frac{A_{4}}{A_{3}}=\frac{\cos \theta_{3} \cos \theta_{0}-\delta \sin ^{2} \theta_{0}}{\cos \theta_{3} \cos \theta_{0}+\delta \sin ^{2} \theta_{0}}, \\
& \frac{A_{6}}{A_{3}}=\frac{\delta \sin 2 \theta_{0}}{\cos \theta_{3} \cos \theta_{0}+\delta \sin ^{2} \theta_{0}} .
\end{aligned}
$$

Equations (2.42) are in complete agreement with the corresponding equations in Achenbach (2001). 
Incident P-wave at isothermal rigid boundary $(h-\infty)$

In this case the amplitude ratio can be calculated as

$$
\begin{aligned}
& \frac{A_{2}}{A_{3}}=\frac{2}{\Delta}\left(\omega^{2}-k_{1}^{2}\right) \frac{k_{3}}{k_{1}} \sin \theta_{0} \sin \theta_{3}, \\
& \frac{A_{4}}{A_{3}}=1-\frac{2}{\Delta} \frac{k_{3}}{k_{1}}\left(\omega^{2}-k_{2}^{2}\right) \sin \theta_{0} \sin \theta_{3}, \\
& \frac{A_{6}}{A_{3}}=-\frac{2}{\Delta} \sin \theta_{0}\left\{\frac{k_{2}}{k_{1}}\left(\omega^{2}-k_{1}^{2}\right) \cos \theta_{2}-\left(\omega^{2}-k_{2}^{2}\right) \cos \theta_{0}\right\}
\end{aligned}
$$

where, $\quad \Delta=\left\{\frac{k_{2}}{k_{1}}\left(\omega^{2}-k_{1}^{2}\right) \sin \theta_{2}-\left(\omega^{2}-k_{2}^{2}\right) \sin \theta_{0}\right\} \frac{k_{3}}{k_{1}} \sin \theta_{3}+$

$$
-\left\{\frac{k_{2}}{k_{1}}\left(\omega^{2}-k_{1}^{2}\right) \cos \theta_{2}-\left(\omega^{2}-k_{2}^{2}\right) \cos \theta_{0}\right\} \frac{k_{3}}{k_{1}} \cos \theta_{3} \text {. }
$$

In the case when $\varepsilon=\tau_{0}=a=0$, the above equations also take the form as in Eq.(2.42).

\section{CASE II: PLANE SV-WAVE INCIDENT UPON THE PLANE SURFACE}

We now proceed to determine the amplitude ratios for an incident SV-wave on the boundary under the same boundary conditions Eq.(2.29).

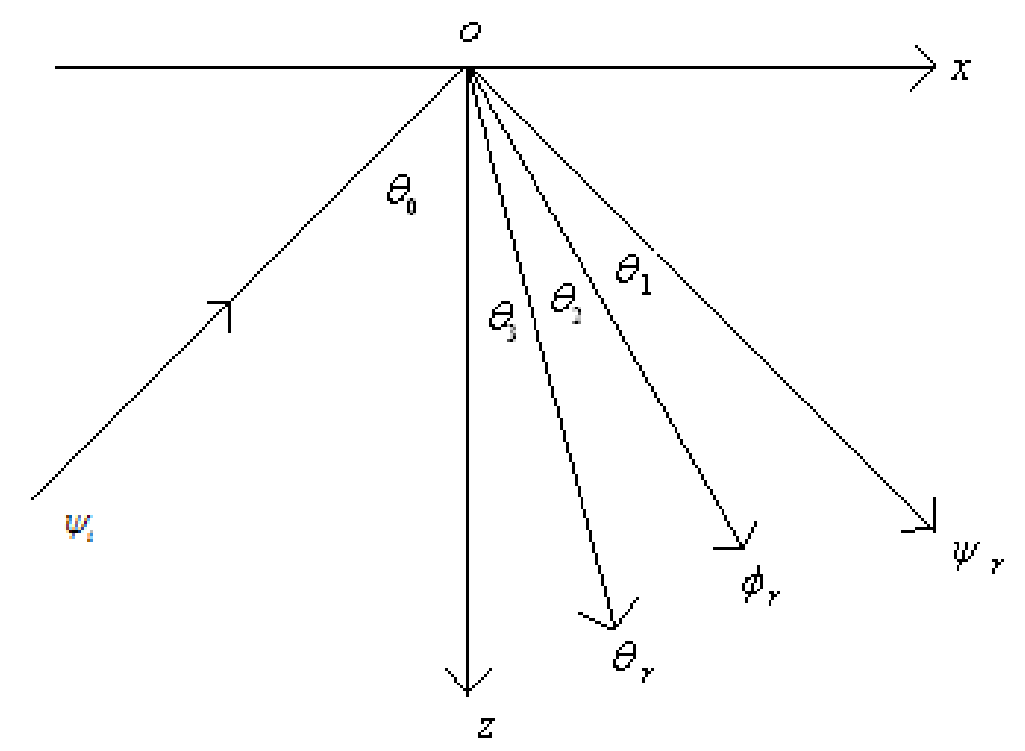

Fig.3. Incident SV-wave and the corresponding reflected waves. 
SV-wave incident upon insulated rigid boundary $(h \rightarrow 0)$

As in Fig.3, we take the incident SV-wave $\psi_{i}$ (omitting the term $e^{-i \omega t}$ ) as

$$
\psi_{i}=A_{5} e^{i k_{3}\left(x \sin \theta_{0}-z \cos \theta_{0}\right)} .
$$

Reflected SV-, P-, and thermal waves may be taken as

$$
\begin{aligned}
& \psi_{r}=A_{6} e^{i k_{3}\left(x \sin \theta_{1}+z \cos \theta_{1}\right)} \\
& \phi_{r}=A_{4} e^{i k_{1}\left(x \sin \theta_{2}+z \cos \theta_{2}\right)}+A_{2} e^{i k_{2}\left(x \sin \theta_{3}+z \cos \theta_{3}\right)} \\
& \left(1-a \nabla^{2}\right) \chi_{r}=b_{2} A_{4} e^{i k_{l}\left(x \sin \theta_{2}+z \cos \theta_{2}\right)}+b_{1} A_{2} e^{i k_{2}\left(x \sin \theta_{3}+z \cos \theta_{3}\right)}
\end{aligned}
$$

where $\quad \psi=\psi_{i}+\psi_{r}, \quad \phi=\phi_{r}, \quad$ and $\quad \chi=\chi_{r}$.

In order to satisfy the boundary conditions Eq.(2.29) when $h \rightarrow 0$, we use Eqs (2.45) and (2.17) in Eq.(2.29) and get

$$
\left[\begin{array}{ccc}
a_{11} & a_{12} & a_{13} \\
a_{21} & a_{22} & a_{23} \\
a_{31} & a_{32} & 0
\end{array}\right]\left[\begin{array}{l}
A_{2} / A_{5} \\
A_{4} / A_{5} \\
A_{6} / A_{5}
\end{array}\right]=\left[\begin{array}{c}
a_{13} \\
-a_{23} \\
0
\end{array}\right]
$$

where

$$
\begin{aligned}
& a_{11}=\frac{k_{2}}{k_{3}} \sin \theta_{3}, \quad a_{12}=\frac{k_{1}}{k_{3}} \sin \theta_{2}, \quad a_{13}=-\cos \theta_{0}, \\
& a_{21}=\frac{k_{2}}{k_{3}} \cos \theta_{3}, \quad a_{22}=\frac{k_{1}}{k_{3}} \cos \theta_{2}, \quad a_{23}=\sin \theta_{0}, \\
& a_{31}=\frac{k_{2}^{3}}{k_{1}^{3}}\left(\frac{\omega^{2}}{k_{2}^{2}}-1\right) \cos \theta_{3}, \quad a_{32}=\left(\frac{\omega^{2}}{k_{1}^{2}}-1\right) \cos \theta_{2} .
\end{aligned}
$$

In this case the following relations corresponding to Eq.(2.33) are obtained

$$
k_{1} \sin \theta_{2}=k_{2} \sin \theta_{3}=k_{3} \sin \theta_{0}=k_{3} \sin \theta_{1} .
$$

Solving the Eqs (2.46), we get

$$
\frac{A_{2}}{A_{5}}=\frac{1}{\Delta}\left(\frac{\omega^{2}}{k_{1}^{2}}-1\right) \cos \theta_{2} \sin 2 \theta_{0},
$$




$$
\begin{aligned}
\frac{A_{4}}{A_{5}} & =-\frac{1}{\Delta} \frac{k_{2}^{3}}{k_{1}^{3}}\left(\frac{\omega^{2}}{k_{2}^{2}}-1\right) \cos \theta_{3} \sin 2 \theta_{0}, \\
& \frac{A_{6}}{A_{3}}=-1-\frac{2}{\Delta} 2 \cos \theta_{0} \cos \theta_{2} \cos \theta_{3}\left\{\frac{k_{2}}{k_{3}}\left(\frac{\omega^{2}}{k_{1}^{2}}-1\right)-\frac{k_{1}}{k_{3}} \frac{k_{2}^{3}}{k_{1}^{3}}\left(\frac{\omega^{2}}{k_{2}^{2}}-1\right)\right\} \\
\text { where, } \quad \Delta & =\left\{\frac{k_{2}}{k_{3}}\left(\frac{\omega^{2}}{k_{1}^{2}}-1\right) \cos \theta_{2} \sin \theta_{3}-\frac{k_{2}^{3}}{k_{1}^{3}} \frac{k_{1}}{k_{3}}\left(\frac{\omega^{2}}{k_{2}^{2}}-1\right) \cos \theta_{3} \sin \theta_{2}\right\} \sin \theta_{0}+ \\
& -\left\{\frac{k_{2}}{k_{3}}\left(\frac{\omega^{2}}{k_{1}^{2}}-1\right) \cos \theta_{3} \cos \theta_{2}-\frac{k_{2}^{3}}{k_{1}^{3}} \frac{k_{1}}{k_{3}}\left(\frac{\omega^{2}}{k_{2}^{2}}-1\right) \cos \theta_{2} \cos \theta_{3}\right\} \cos \theta_{0} .
\end{aligned}
$$

In the case when $\varepsilon=\tau_{0}=a=0$, Eq.(2.48) takes the form

$$
\begin{aligned}
& \frac{A_{2}}{A_{5}}=0, \quad \frac{A_{4}}{A_{5}}=\frac{\sin 2 \theta_{0}}{\delta \cos \theta_{2} \cos \theta_{0}+\sin ^{2} \theta_{0}}, \\
& \frac{A_{6}}{A_{5}}=\frac{\delta \cos \theta_{2} \cos \theta_{0}-\sin ^{2} \theta_{0}}{\delta \cos \theta_{2} \cos \theta_{0}+\sin ^{2} \theta_{0}}
\end{aligned}
$$

Equations (2.49) are in complete agreement with the corresponding equations in Achenbach (2001).

\section{SV-wave incident upon isothermal rigid boundary $(h \rightarrow \infty)$}

We follow a similar procedure as in the earlier sections to calculate the amplitude ratios. Equations (2.45), (2.46) along with the boundary conditions Eq.(2.29) are used when $h \rightarrow \infty$. The ratio becomes

$$
\begin{aligned}
& \frac{A_{2}}{A_{5}}=\frac{1}{\Delta}\left(\omega^{2}-k_{1}^{2}\right) \sin 2 \theta_{0}, \\
& \frac{A_{4}}{A_{5}}=-\frac{1}{\Delta}\left(\omega^{2}-k_{2}^{2}\right) \sin 2 \theta_{0}, \\
& \frac{A_{6}}{A_{5}}=-1-\frac{2}{\Delta} \cos \theta_{0}\left\{\frac{k_{2}}{k_{3}}\left(\omega^{2}-k_{1}^{2}\right) \cos \theta_{3}-\frac{k_{1}}{k_{3}}\left(\omega^{2}-k_{2}^{2}\right) \cos \theta_{2}\right\} \\
& \Delta=\left\{\frac{k_{2}}{k_{3}}\left(\omega^{2}-k_{1}^{2}\right) \sin \theta_{3}-\frac{k_{1}}{k_{3}}\left(\omega^{2}-k_{2}^{2}\right) \sin \theta_{2}\right\} \sin \theta_{0}+ \\
& -\left\{\frac{k_{2}}{k_{3}}\left(\omega^{2}-k_{1}^{2}\right) \cos \theta_{3}-\frac{k_{1}}{k_{3}}\left(\omega^{2}-k_{2}^{2}\right) \cos \theta_{2}\right\} \cos \theta_{0} .
\end{aligned}
$$

In the case when $\varepsilon=\tau_{0}=a=0$, the above Eq.(2.50) becomes identical with Eqs (2.49). 


\section{Some observations of the results}

Thus we see from the above results that the amplitude ratios depend on the initial angle of reflection $\theta_{0}$, coupling factor $\varepsilon$, relaxation time parameter $\tau$, the two temperature parameter $a$ and the frequency $\omega$. We now analyze our results for different cases. Since the amplitude ratios are complex quantities, in the following sections, the amplitude ratios of the form $\frac{A_{2}}{A_{3}}$ will be considered as $\left|\frac{A_{2}}{A_{3}}\right|$. For illustrating and comparing the results of the two temperature LS-model with the classical coupled thermoelasticity (CCTE) model, we choose a copper material for which the parameters are as follows

$$
\begin{aligned}
& \lambda=7.76 \times(10)^{10} \mathrm{~N} / \mathrm{m}^{2}, \quad \mu=3.86 \times(10)^{10} \mathrm{~N} / \mathrm{m}^{2}, \quad \rho=8,954 \mathrm{~kg} / \mathrm{m}^{3}, \\
& \alpha_{t}=1.78 \times(10)^{-5} \mathrm{deg}^{-1}, \quad c_{E}=383.1 \mathrm{~J} \mathrm{~kg}^{-1} \mathrm{deg}^{-1}, \quad \varepsilon=0.0168, \\
& k=386 \mathrm{Wm}^{-1} \mathrm{deg}^{-1}, \quad T_{0}=293 \mathrm{~K}, \quad \tau_{0}=0.018766, \\
& a=0.016, \quad \delta^{2}=0.2494 .
\end{aligned}
$$

\section{A. Reflection of P-waves on rigid insulated boundary}

(i) From the nature of the graph for the amplitude ratio $\frac{A_{2}}{A_{3}}$ in $0<\theta_{0}<90^{\circ}$, we notice that for each value of the frequency $\omega$ in the range $0<\omega \leq 7.5$, the maximum value increases from 0.01296 to 0.4926 when the initial angle of incidence $\theta_{0} \approx 45.85^{\circ}, a \neq 0, \varepsilon \neq 0, \tau_{0} \neq 0$ (LS-model). Only a small change of value 0.4806 occurs for the maximum when $a \neq 0, \varepsilon \neq 0, \tau_{0}=0$ (CCTE model). We further notice that at about $\omega=10.0$ there are two distinct maxima for the ratio $\frac{A_{2}}{A_{3}}$, one at $\theta_{0}=29.24^{0}$ and the other at $\theta_{0}=76.82^{\circ}$ with values 0.4902 and 0.1687 , respectively. The minimum value is recorded at 0.06129 at $\theta_{0}=61.91^{0}$. The graphs are plotted for $\omega=9$ and $\omega=10$ in Fig.4.

(ii) For the two temperature LS-model $\left(a \neq 0, \varepsilon \neq 0, \tau_{0} \neq 0\right)$ and two temperature CCTE model $\left(a \neq 0, \varepsilon \neq 0, \tau_{0}=0\right)$ the graphs for $\frac{A_{2}}{A_{3}}$ have been depicted in Fig.5 for $\omega=1.75$ in the range $0<\theta_{0}<90^{\circ}$. In order to compute the results with the corresponding cases when $a=0$ (no conductive temperature) two other graphs have been drawn in Fig.5. It is seen that the maximum value of $\frac{A_{2}}{A_{3}}$ ranges from 0.01005 to 0.05019 . Thus the conductive temperature has a significant effect on the magnitude of $\frac{A_{2}}{A_{3}}$ for these chosen parameters. 


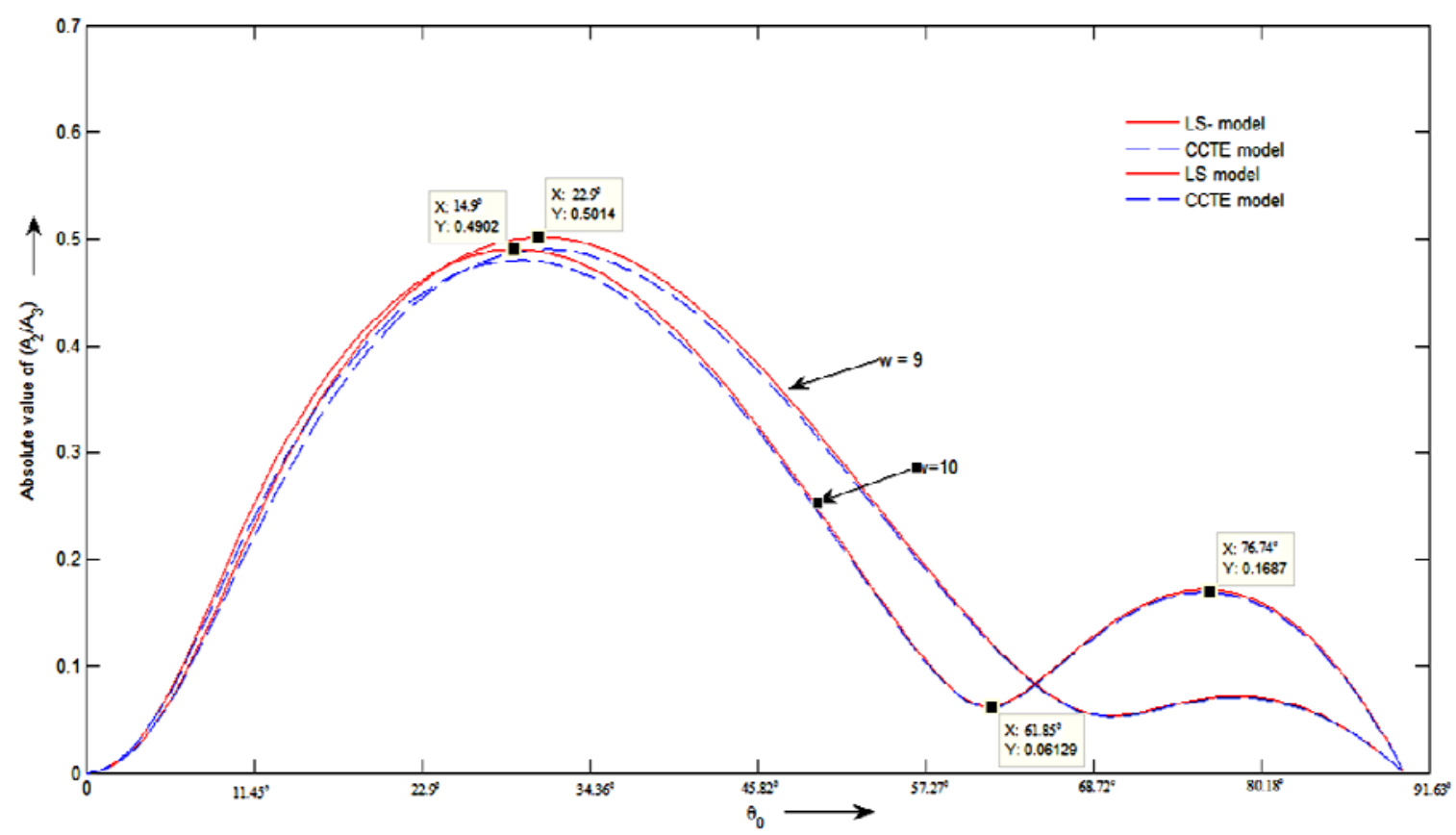

Fig.4. Variation of $\frac{A_{2}}{A_{3}}$ for incident P-wave for insulated boundary.

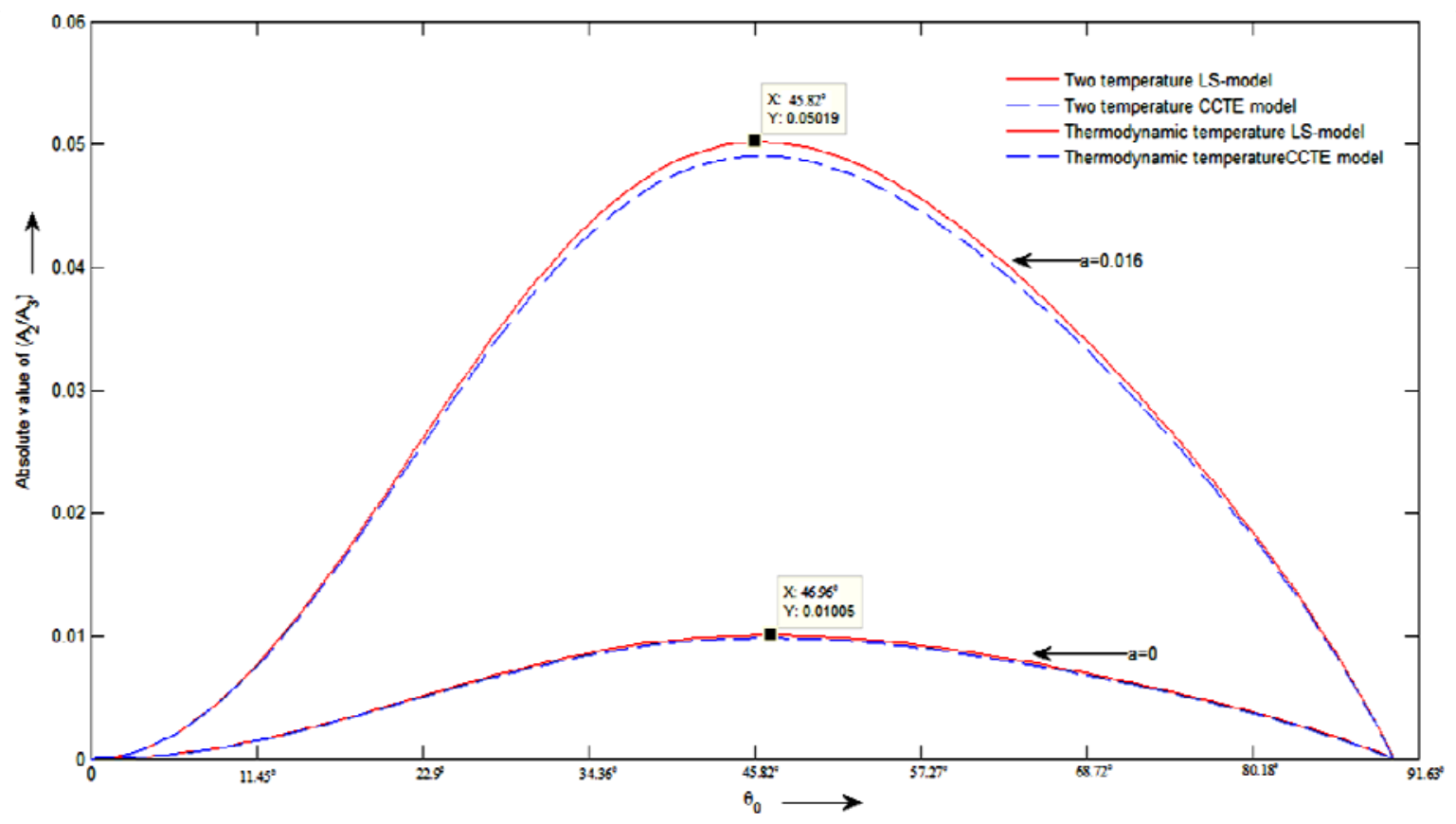

Fig.5. Variation of $\frac{A_{2}}{A_{3}}$ for incident P-wave for insulated boundary.

(iii) Figure 6 shows that for normal incidence $\left(\theta_{0}=0\right)$ the amplitude of the reflected wave $A_{4}$ is equal to the amplitude of the incident wave $A_{3}$. In the range $0<\theta_{0}<63^{0}$, the ratio $\frac{A_{4}}{A_{3}}$ decreases to zero from unity, but the ratio reverses during $63^{\circ}<\theta_{0}<90^{\circ}$ when the ratio $\frac{A_{4}}{A_{3}}$ assumes zero at $\theta_{0}=63^{0}$ and 
unity at $\theta_{0} \approx 90^{\circ}$. It is further observed from the graphs that the L-S two temperature, L-S CCTE and two temperature CCTE models yield almost identical results.

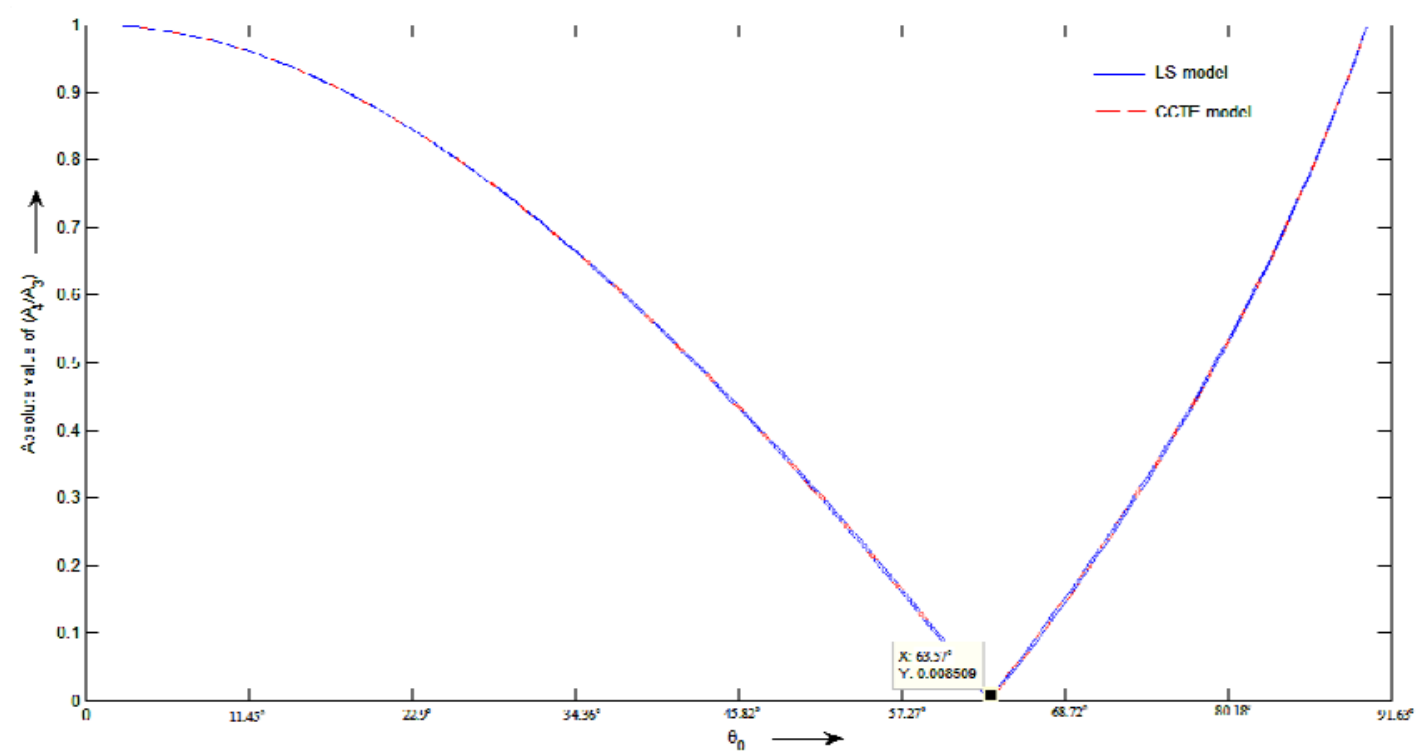

Fig.6. Variation of $\frac{A_{4}}{A_{3}}$ for incident P-wave for insulated boundary.

(iv) The graphs of the thermodynamic temperature L-S model and two temperature L-S models for $\frac{A_{6}}{A_{3}}$ have been presented in Fig.7. It is noticed that in both cases the ratio $\frac{A_{6}}{A_{3}}$ assumes almost identical values. The ratio starts with the zero value at normal incidence and increases to a maximum of 0.5591 at $\theta_{0}=49.87^{\circ}$; thereafter it decreases to zero as $\theta_{0}$ increases in $49.87<\theta_{0}<90^{\circ}$.

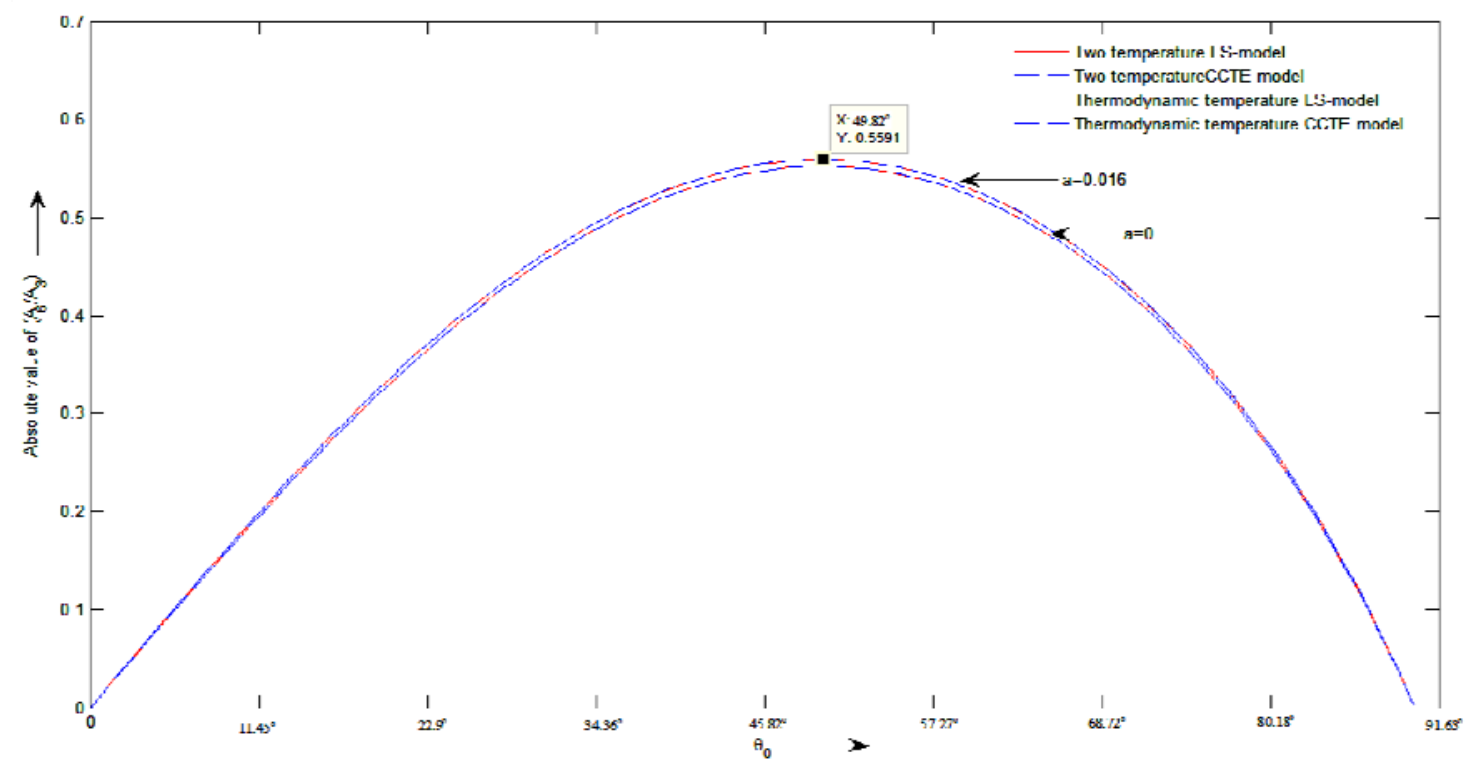

Fig.7. Variation of $\frac{A_{6}}{A_{3}}$ for incident P-wave for insulated boundary. 


\section{B. Reflection of P-waves on rigid isothermal boundary}

(i) Several graphs of $\frac{A_{2}}{A_{3}}$ in this case have been drawn for values of $\omega=10$ to $\omega=35$ in the range $0<\theta_{0}<90^{\circ}$. It is observed that for $\omega=35$, the ratio assumes the zero value at normal incidence and then sharply increases to a maximum of 1.719 at $\theta_{0}=25.79^{\circ}$. A similar graph has been predicted for $\omega=20$ with a peak value of 1.534 at $\theta_{0}=45.86^{\circ}$. In the case when $\omega=10$, the ratio assumes the zero value at normal incidence but assumes no peak value in $0<\theta_{0}<90^{\circ}$. In all the three cases mentioned above, the ratio $\frac{A_{2}}{A_{3}}$ converges to unity at about $\theta_{0}=90^{\circ}$ (see Fig.8). It is noticed that the two temperature parameter $a$ is not significant in this case.

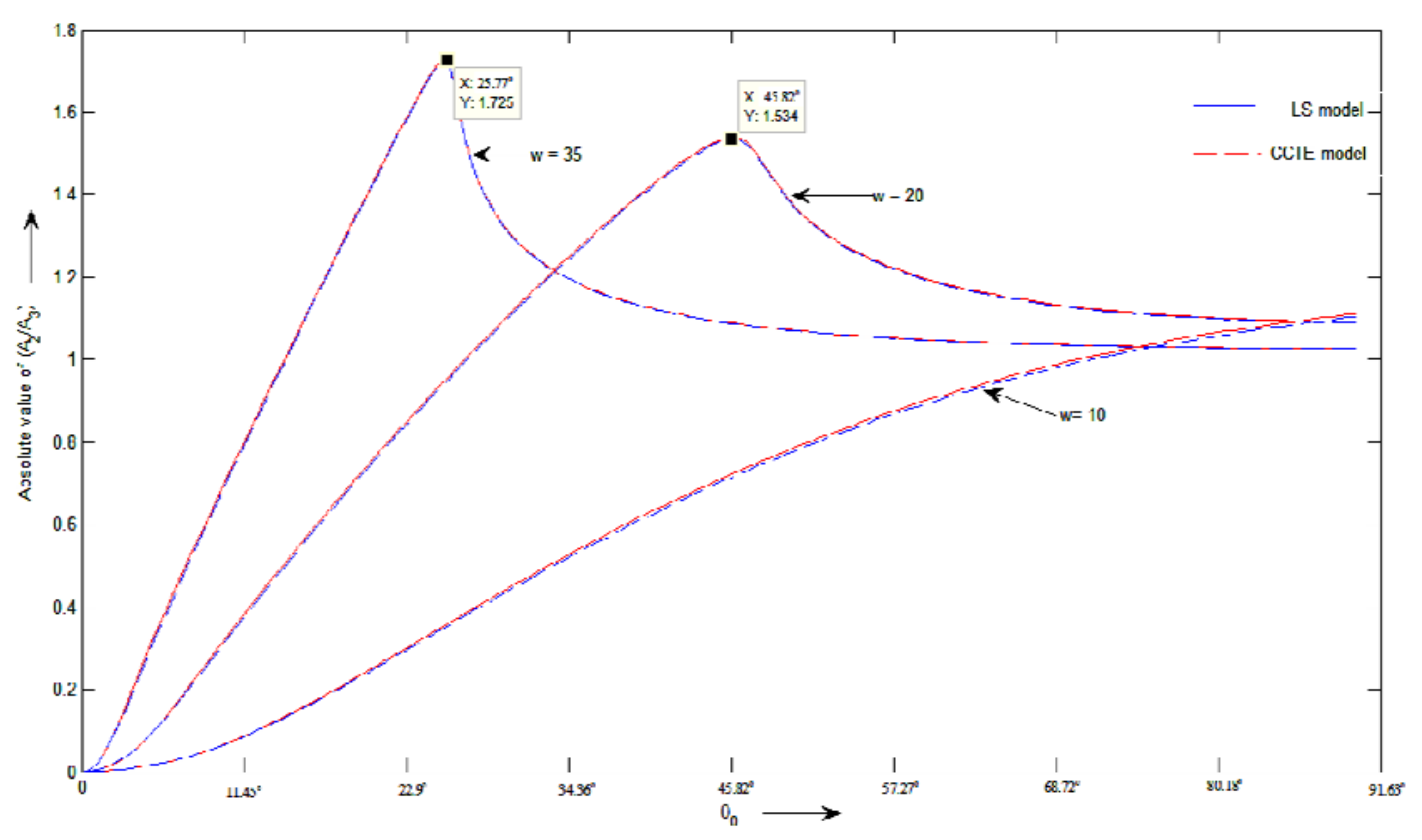

Fig.8. Variation of $\frac{A_{2}}{A_{3}}$ for incident P-wave for isothermal boundary.

(ii) From Fig.9 we see that the two temperature L-S model and thermodynamic L-S model start with the unity value and then both decrease with nearly equal values to a minimum of 0.1032 at $66.497^{0}$ and 0.06582 at $67.64^{\circ}$, respectively. Thereafter, significant changes in values are observed in the ratio $\frac{A_{4}}{A_{3}}$ which raises to nearly 0.6297 before termination in the range $66.497^{0}<\theta_{0}<90^{0}$. 


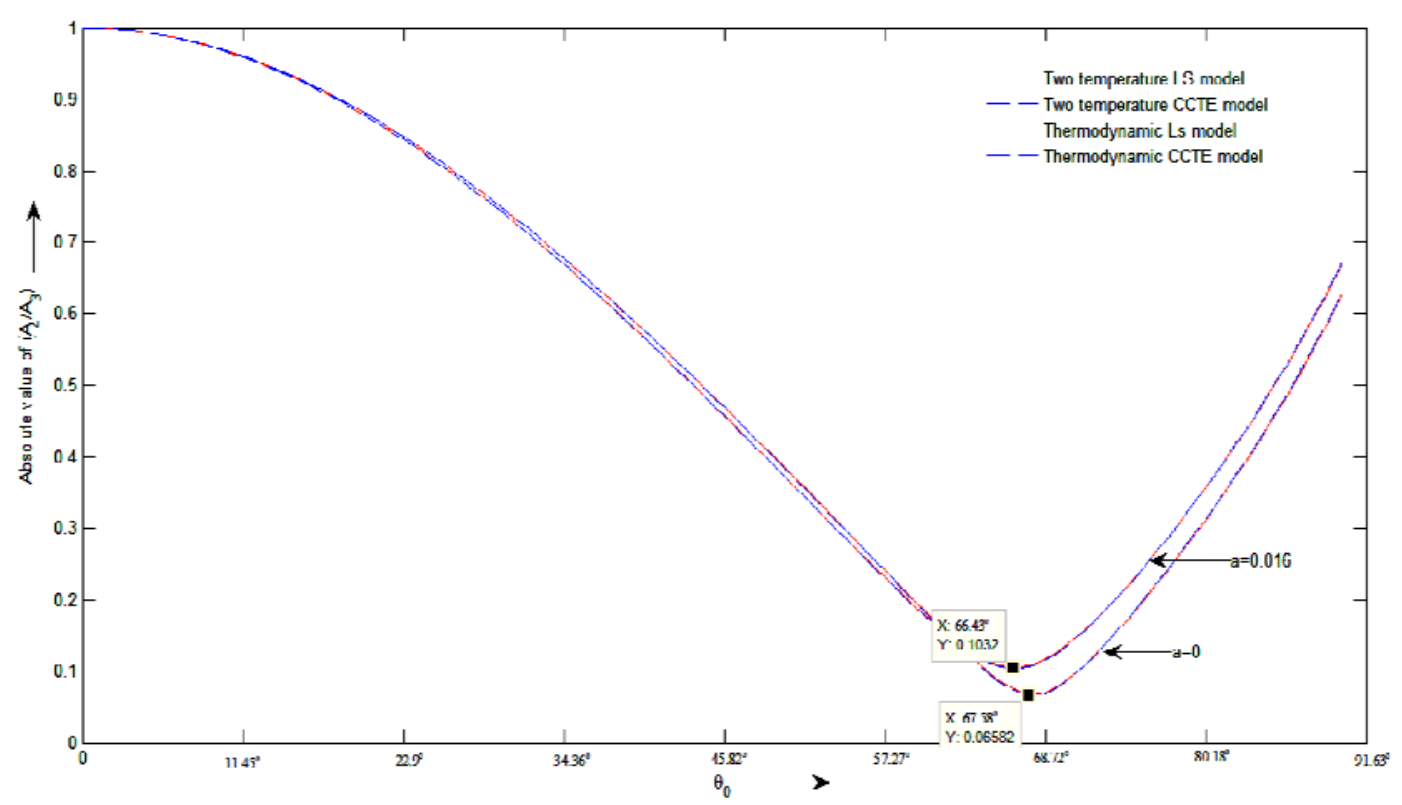

Fig.9. Variation of $\frac{A_{4}}{A_{3}}$ for incident P-wave for isothermal boundary.

(iii) It is interesting to note the role of the two temperature parameter in the graph of $\frac{A_{6}}{A_{3}}$ in Fig.10, when $\omega=1.75$. The parameter does not make any significant change in values until the graphs raise from zero to 0.512 as maximum at $\theta_{0}=46.43^{\circ}$. Thereafter, significant changes in values are observed in $46.43^{\circ}<\theta_{0}<90^{\circ}$.

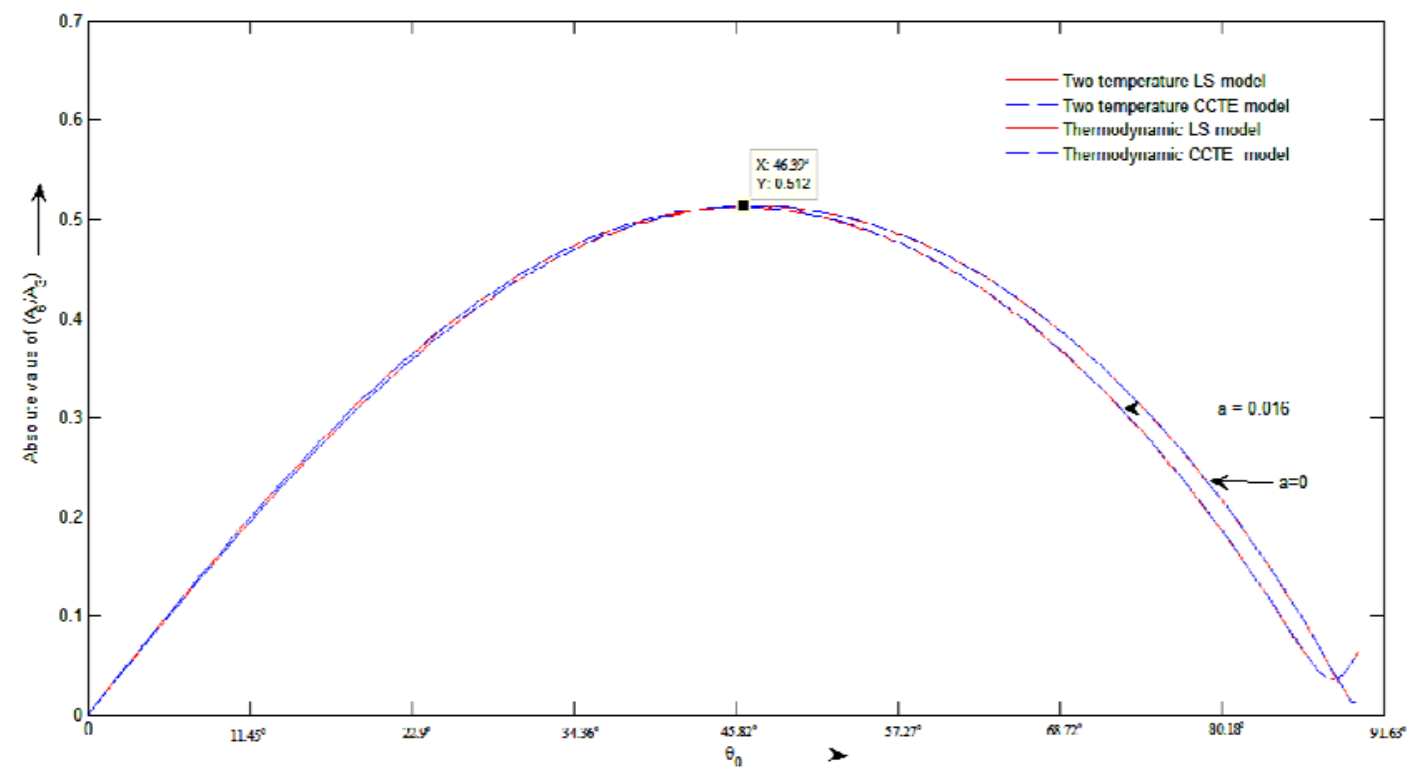

Fig.10. Variation of $\frac{A_{6}}{A_{3}}$ for incident P-wave for isothermal boundary. 


\section{Reflection of SV-waves on rigid insulated boundary}

(i) Figure 11 depicts graphs of $\frac{A_{2}}{A_{5}}$ incorporating the two temperature models on (a) LS and (b) CCTE and also dynamic temperature models on (c) LS and (d) CCTE. It is observed that the two temperature parameter ' $a$ ' is very significant in the sense that the conductive temperature tends to lower the maximum values $0.6138 \times(10)^{-2}$ and $0.608 \times(10)^{-2}$, respectively, to $0.3963 \times(10)^{-2}$ and $0.3949 \times(10)^{-2}$ at $\theta_{0}=16.28^{0}$ and $\theta_{0}=46.15^{\circ}$, respectively in both cases. The minimum value of $1.215 \times(10)^{-3}$ has been diminished to $0.556 \times(10)^{-3}$ at about $\theta_{0}=29.8^{0}$. It is further observed that the relaxation time parameter $\tau_{0}$ has contributed only to a little change in values towards the neighborhood of the two peaks. In all the cases the ratio $\frac{A_{2}}{A_{5}}$ tends to zero as $\theta_{0}$ increases from $29.8^{\circ}$ to $90^{\circ}$.

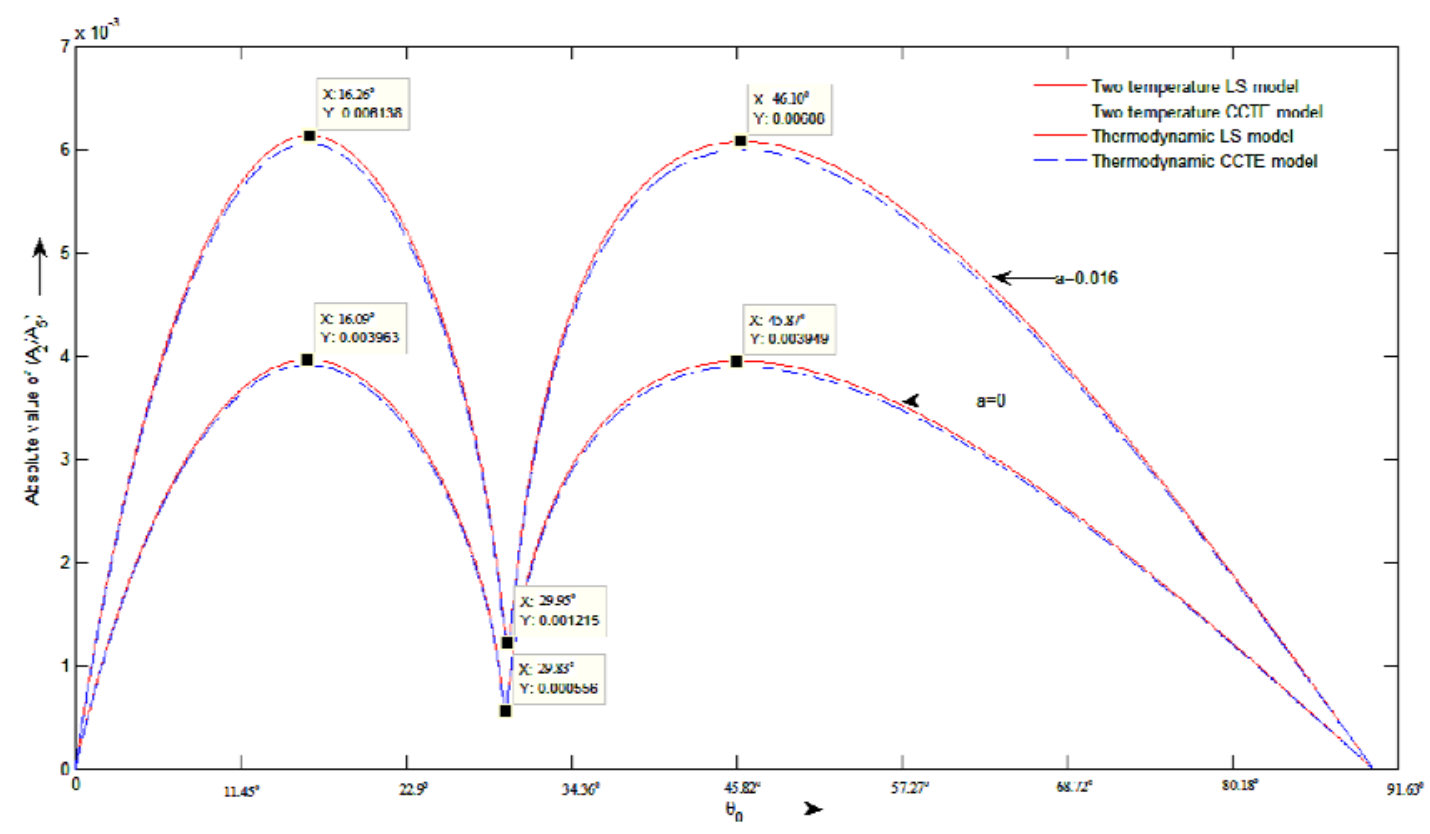

Fig.11. Variation of $\frac{A_{2}}{A_{5}}$ for incident SV-wave for insulated boundary.

(ii) A graph (not shown) of $\frac{A_{4}}{A_{5}}$ has been drawn for increasing $\theta_{0}$ and it has been noticed that the ratio steadily increases to a maximum of $48.42^{\circ}$ and $30.57^{\circ}$ and then decreases to zero as $\theta_{0}$ increases further. The parameters $a$ and $\tau_{0}$ are almost insignificant in this case.

(iii) The nature of the graph for the ratio $\frac{A_{6}}{A_{5}}$ is interesting in the sense that at normal incidence the amplitude of the incident and reflected SV-wave $A_{5}$ and $A_{6}$ respectively becomes equal. Again in the 
range $33.25^{\circ}<\theta_{0}<90^{\circ}$ the amplitudes are equal. Further, a sharp decrease has been observed in the range $0<\theta_{0}<12.38^{0}$. Thereafter the ratio $\frac{A_{6}}{A_{5}}$ steadily increases to unity as shown in Fig. 12 .

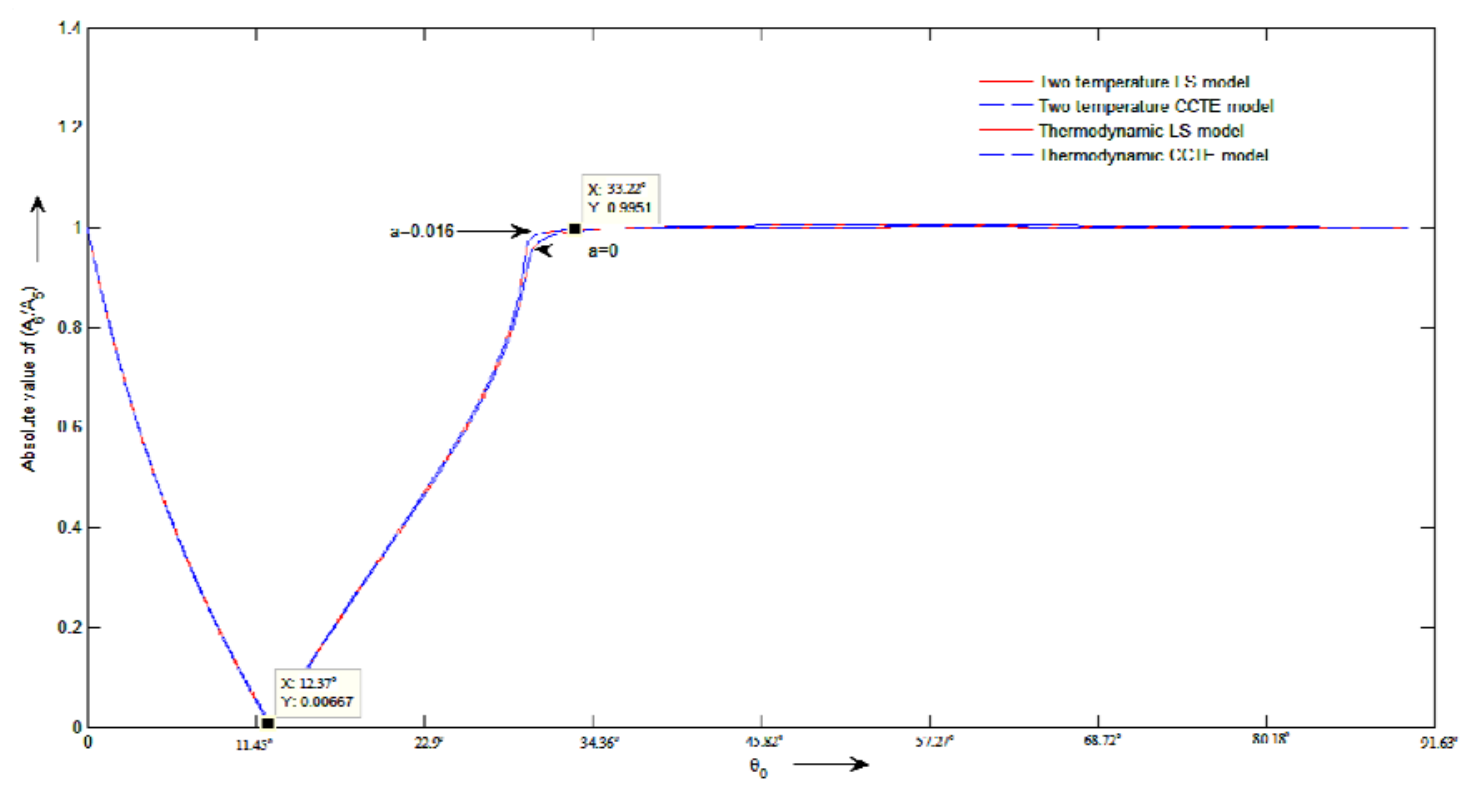

Fig.12. Variation of $\frac{A_{6}}{A_{5}}$ for incident SV-wave for insulated boundary.

\section{Reflection of SV-waves on rigid isothermal boundary}

(i) In Fig.13, significant changes have been observed in the values of $\frac{A_{2}}{A_{5}}$ due to the two temperature parameter. The values of the graph for the two temperature LS-model and dynamical CCTE model steadily increase from zero to the maximum of 0.03886 and 0.02732 respectively at about $\theta_{0} \approx 29.8^{0}$. They graphs go to zero as $\theta_{0}$ increases in the range $30.33^{\circ}<\theta_{0}<90^{\circ}$. In this case also, the relaxation constant create insignificant changes in the values of the ratio $\frac{A_{2}}{A_{5}}$.

(ii) In the graph of $\frac{A_{4}}{A_{5}}$ (not shown) it is noticed that the nature of the graph is almost the same as observed in the former case D (i) (Fig.13) for $\frac{A_{2}}{A_{5}}$ except the fact that for all cases (a), (b), (c), (d) as mentioned in section $\mathrm{C}$ (i) the graphs almost coincide.

(iii) The nature of the graph of $\frac{A_{6}}{A_{5}}$ is almost similar to that of $\frac{A_{6}}{A_{5}}$ in the case of insulated boundary (Fig.12 as explained in section $\mathrm{C}$ (iii)). The difference observed in this case is that the ratio reaches zero from unity at $\theta_{0} \approx 24.65^{\circ}$ but returns to unity almost vertically at about $\theta_{0} \approx 30.38^{\circ}$. 


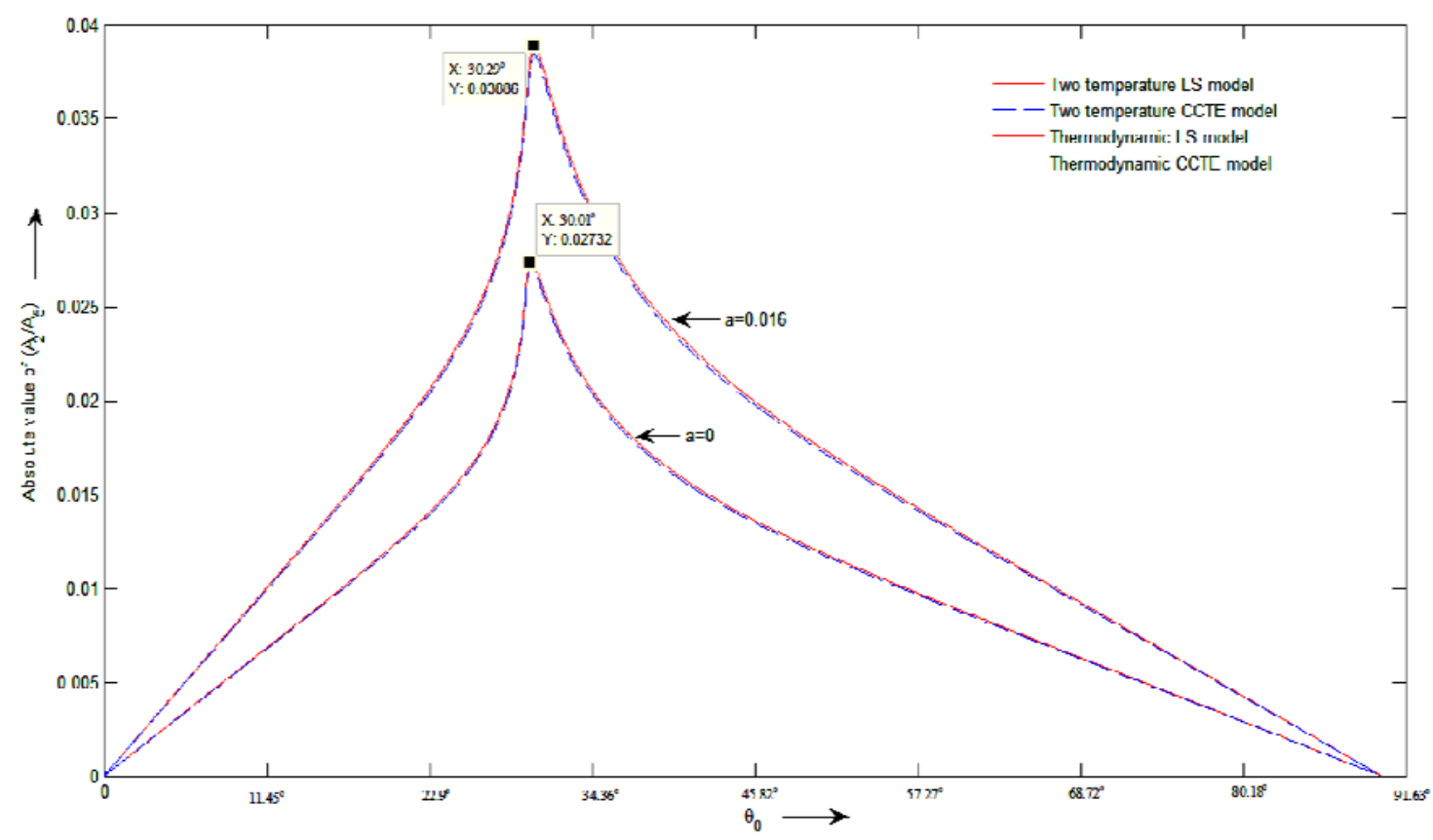

Fig.13. Variation of $\frac{A_{2}}{A_{5}}$ for incident SV-wave for isothermal boundary.

\section{Acknowledgement}

The authors are thankful to Prof. B.P. Chattopadhyay, Principal, Brainware College of Engineering for his constant encouragement in the preparation of this paper.

\section{Nomenclature}

$$
\begin{aligned}
a & - \text { two temperature parameter } \\
c_{E} & - \text { specific heat at constant strain } \\
K & - \text { thermal conductivity } \\
k & - \text { wave number } \\
T_{0} & - \text { the reference temperature } \\
\alpha_{t}=\frac{\gamma}{(3 \lambda+2 \mu)} & - \text { coefficient of thermal expansion } \\
\lambda \text { and } \mu & - \text { Lame constants } \\
\rho & - \text { mass density } \\
\tau_{0} & - \text { relaxation time parameter } \\
\phi \text { and } \psi & - \text { thermoelastic potential functions } \\
\omega & - \text { circular frequency of wave }
\end{aligned}
$$

\section{References}

Achenbach J.D. (2001): Wave Propagation in Elastic Solids. - Amsterdam: North Holland Pub. Co.

Bassiouny E. and Youssef H.M. (2008): Two-temperature generalized thermoelasticity of finite rod subjected to different types of thermal loading. - Journal of Thermal Stresses, vol.31, pp.233-245. 
Chen P.J., Gurtin M.E. and Williams W.O. (1968): On a theory of heat conduction involving two temperatures. - Z. Angew. Math. Phys., vol.19, pp.614-627.

Chen P.J., Gurtin M.E. and Williams W.O. (1968): On the thermodynamics of non-simple elastic material with two temperatures. - Z. Angew. Math. Phys., vol.20, pp.107-112.

Das N.C., Lahiri A., Sarkar S., and Basu S. (2008): Reflection of generalized thermoelastic waves from isothermal and insulated boundaries of a half space. - Department of Mathematics. Jadavpur University. Computers and Mathematics with Applications, vol.56, pp.2795-2805.

Green A.E. and Lindsay K.A. (1972): Thermoelasticity. - J. Elasticity, vol.2, pp.1-7.

Green A.E. and Naghdi P.M. (1992): On undamped heat waves in an elastic solid. - J. Thermal Stresses, vol.15, pp.252-264.

Green A.E. and Naghdi P.M. (1993): Thermoelasticity without energy dissipation. - J. Elasticity, vol.31, pp.189-208.

Green A.E. and Naghdi P.M. (1996): Thermoelasticity without energy dissipation. - Jour. Therm. Stress. vol.19, pp.267-272.

Lord H. and Shulman Y. (1967): A generalized dynamic theory of thermoelasticity. - J. Mech. Phys. Solids, vol.15, pp.299-309.

Suhubi E.S. (1982): A generalized theory on simple thermomechanical materials. - Int. J. Engg. Sci., vol.20, pp.367371.

Youssef H.M. (2006): On a theory of two temperature-generalized thermoelasticity. - IMA J. Appl. Math. Phys., vol.71, pp.383-390.

Youssef H.M. and Al-Harby A. (2007): On the theory of state-space approach of two-temperature generalized thermoelasticity of infinite body with a spherical cavity subjected to different types of thermal loading. - Arc. Appl. Mech., vol.77, pp.675-687. 